of the word; those selected are, however, sufficiently closely associated with melanomas that they should be further characterized and serious consideration given to their potential as in vitro and in vivo markers for diagnosis and as targets for immunotherapy. Simple screening of monoclonal antibodies by binding assays with cultured tumour cell lines is not sufficient to evaluate critically their specificity, and screening of a large variety of both normal and tumour tissues by indirect immunofluorescence or immunoperoxidase techniques appears necessary to make any meaningful comparisons. Quantification of antigen expression on normal and tumour cells is definitely necessary, either by saturation binding with Scatchard plot analyses or by quantitative binding assays with radiolabelled antibody or by analysis with the fluorescence-activated cell sorter.

\title{
What limits photosynthesis?
}

\section{from Christine Foyer, Richard Leegood and David Walker}

ENHANCED plant productivity is one means by which world food and energy shortages may be alleviated. For this reason research into the photosynthetic process is increasingly favoured both in the UK and overseas. However, the crop physiologist is quick to remind us that the connection between photosynthesis and productivity is more apparent than real, in the sense that a correlation between short-term measurement of photosynthetic rate and crop yield is not easily demonstrated. Certainly the gulf between the plant scientist and the farmer is considerable. Even the highly valuable contributions of the plant breeder have been largely confined to factors such as disease resistance and the redistribution of assimilates within the plant. We have all lamented photorespiratory loss, worried over the inadequacies of ribulose bisphosphate carboxylase/oxygenase and wondered if we can turn, for solace, to $\mathrm{C} 4$. Perhaps, however, if we pause for thought, there is no reason to be unduly pessimistic about the future of photosynthesis in its relation to agriculture.

Plant growth is the best measure of net photosynthesis that we have and the limits to which the useful redistribution of assimilates can be achieved are fast being reached. To increase plant productivity by securing more vigorous growth through more effective photosynthesis we need to determine which existing limitation might be removed. A discussion meeting* was organized by the Agricultural Research Council to identify and characterize the reactions which limit the photosynthetic process in various environmental and developmental conditions, and to determine how basic research into photosynthesis may be directed towards increasing plant productivity. A workshop on the methodology and applications of the measurement of chlorophyll fluorescence preceded the meeting. This technique is now regarded as a valuable non-intrusive probe for photosynthesis in vivo providing, in the words of $\mathrm{J}$. Barber *The ARC discussion meeting on 'What limits photosynthesis' was held on 15-16 April 1982 and was hosted by the ARC Sheffield.
(Imperial College, London), a 'stethoscope' for plants.

Many workers in photosynthesis disagree about the basis on which the rate of photosynthesis should be expressed, and doubt the validity of the present methods, especially since a single factor is unlikely to be constant. All quantitative means for expressing photosynthetic rate in current use (for example, ground area, fresh weight) carry inescapable disadvantages. In spite of this, chlorophyll is likely to remain the universal basis for expressing photosynthetic rate at the cellular level simply because it is easily measured ( $\mathrm{J}$. Ludwig, GCRI, Littlehampton). The use of the term 'photosynthetic efficacy', to relate capacity to performance (that is, an assessment of the actual performance of a given crop as a function of the potential performance), could help to determine the degree to which photosynthetic capacity is realized in a given circumstance (D. A. Walker, Sheffield University).

Although the basic climatic limitation to crop photosynthesis is the seasonal input of light energy, the degree to which this energy can be used by the plant is modified by local climatic factors, such as temperature, water and the requirements of particular farming systems (J. P. Cooper, WPBI, Aberystwyth). The rate of photosynthesis reaches a maximum at or just before full leaf expansion and declines thereafter. The length of the growing season before leaf senescence is, therefore, a limitation on crop yield (H. Thomas, WPBI, Aberystwyth). The efficiency of photosynthesis in relation to the balanced excitation of the photosystems is believed to be controlled by the phosphorylation of the lightharvesting chlorophyll $a / b$ binding protein. Balanced excitation is necessary for efficient non-cyclic electron transport and also serves to poise cyclic electron flow by increasing excitation of photosystem I relative to photosystem II. Furthermore, the flexible distribution of available quanta between the two photosystems can be especially important under stress and allow a more efficient distribution of excitation in different cells of a leaf and different leaves in a canopy (P. Horton, Sheffield University).

The majority of temperature crops, particularly cereals, are exposed to suboptimal temperatures for most of their lives. Dramatic differences in temperature response occur between cold-tolerant and cold-intolerant plants. Even at the level of the chloroplast the partitioning of assimilate may be considerably influenced by temperature as transport generally outstrips production at low temperatures; the autocatalytic Benson-Calvin Cycle does not obey Arrhenius' law (Heber, Würzburg University). Low temperatures cause phase changes in the lipids of the thylakoid membranes and the resulting reduction of the fluidity of the thylakoids reduces the mobility of the electron carriers between photosystems I and II, for example by diminishing the diffusion rate of reduced plastoquinone (D. Chapman, Imperial College, London). This may well be the most temperature-dependent step of electron transport.

Ribulose bisphosphate carboxylase/ oxygenase is the point of entry of carbon into the Benson-Calvin cycle and that of oxygen into the photorespiratory pathway. There is a broad correlation between ribulose bisphosphate carboxylase activity extractable from leaves and light-saturated rates of photosynthesis in plants taken from a wide range of growth conditions and genotypes. Ribulose bisphosphate carboxylase appears to limit photosynthesis only in the sense that the activity of the enzyme is close to that of the flux through the Benson-Calvin cycle. Plants synthesize only as much of this enzyme as they need (A. Keys, Rothamsted Experimental Station). This raises the question of whether a limitation imposed by this enzyme is a phenomenon perceived by the manipulator rather than the plant.

The purpose of photorespiration continues to be hotly debated. Selective inhibition of the oxygenase activity is improbable although some species have carboxylases with a significantly higher affinity for $\mathrm{CO}_{2}$ than others (S. Gutteridge, Rothamsted Experimental Station). Other enzymes of the Benson-Calvin cycle, such as fructose bisphosphatase, show changes in activity in response to the redox state of ferredoxin, although the changes in fructose bisphosphatase activity do not have a role in regulation of the photosynthetic rate in leaves illuminated with saturating light.

No final answer as to "What limits photosynthesis' arose from the meeting. The most popular candidate, proposed by O. Björkman (Carnegie Institute of Washington), was God. The question deserves future consideration and research if we are to secure increased plant productivity.

Christine Foyer, Richard Leegood and David Walker are in the $A R C$ Research Group on Photosynthesis, Department of Botany, Sheffied S1O 2TN. 